\title{
BMJ Open Poor treatment outcomes of children on highly active antiretroviral therapy: protocol for a systematic review and meta-analysis
}

Kendalem Asmare Atalell (D) , ${ }^{1}$ Kefyalew Addis Alene (iD) ${ }^{2,3}$

To cite: Atalell KA, Alene KA. Poor treatment outcomes of children on highly active antiretroviral therapy: protocol for a systematic review and meta-analysis. BMJ Open 2020;10:e040161. doi:10.1136/ bmjopen-2020-040161

- Prepublication history for this paper is available online. To view these files, please visit the journal online (http://dx.doi. org/10.1136/bmjopen-2020040161).

Received 11 May 2020 Revised 05 November 2020 Accepted 19 November 2020

Check for updates

(C) Author(s) (or their employer(s)) 2020. Re-use permitted under CC BY-NC. No commercial re-use. See rights and permissions. Published by BMJ.

${ }^{1}$ Department of Pediatrics and Child Health Nursing, School of Nursing,College of Medicine and Health Sciences, University of Gondar, Gondar, Amhara, Ethiopia

${ }^{2}$ Faculty of Health Sciences, Curtin University, Bentley Campus, Perth, Western Australia, Australia

${ }^{3}$ Wesfarmers Centre of Vaccines and Infectious Diseases, Telethon Kids Institute, Perth, Western Australia, Australia

Correspondence to Mr Kendalem Asmare Atalell; kedasmar@gmail.com

\section{ABSTRACT}

Introduction While access to highly active antiretroviral therapy (HAART) for children with HIV has expanded and the use of HAART has substantially reduced the morbidity and mortality of children due to HIV, poor treatment outcomes among children with HIV are still a major public health problem globally. The aim of this systematic review and meta-analysis is to quantify treatment outcomes among children with HIV.

Methods and analysis Systematic searches will be conducted in three electronic databases (PubMed, SCOPUS and Web of Science) for recent studies published from 01 Jan 2000 up to 28 October 2020, without geographical restriction. The primary outcomes of the study will be poor treatment outcomes, which include death, treatment failure and loss to follow-up. We will include quantitative studies that report treatment outcomes among children under the age of 18 years with HIV. Studies will be excluded if they are case report, case series, conducted among adults only or do not provide data on treatment outcomes for children. Two researchers will screen the titles and abstracts of all citations identified in our search, then review the full text of the remaining papers to identify those that meet the inclusion criteria. The NewcastleOttawa Scale will be used for quality assessment. A random-effects meta-analysis will be used to obtain pooled estimates of the proportion of poor treatment outcomes. The heterogeneity between studies will be checked visually by using forest plots and quantitatively measured by the index of heterogeneity $\left(\mathrm{I}^{2}\right)$. Pooled estimates of poor treatment outcomes will be calculated with a random-effects model. Subgroup analysis will be conducted by study settings, treatment regimen, comorbidity (such as tuberculosis), study period and HIV type (HIV-1 and HIV-2).

Ethics and dissemination Ethical approval will not be required for this study as it will be based on published papers. The final report of this review will be published in a peer-reviewed scientific journal.

\section{INTRODUCTION}

HIV is the second leading cause of death (after tuberculosis/TB) among infectious diseases, killing more than 1 million people every year and 35.4 million people since the start of the epidemic. ${ }^{2}{ }^{2}$ Although HIV is
Strengths and limitations of this study

- This systematic review will be the first to synthesise treatment outcomes among children with HIV.

- The search will be conducted without geographical restrictions.

- The data reporting will adhere to the Preferred Reporting Items for Systematic reviews and MetaAnalyses guidelines.

- A potential limitation of this study will be a large degree of heterogeneity between published studies.

- The systematic review will be limited to studies published in English language only.

more common among sexually active people, it also affects vulnerable groups, including children and youth. According to the recent Joint United Nations Programme on HIV/ AIDS' (UNAIDS) 2019 Global Report, 1.7 million children younger than 15 years were estimated to be living with HIV, of whom 160 000 were newly infected in 2018 and 110000 children died from AIDS-related illness in the same year. ${ }^{12}$ More than $90 \%$ of these children were living in sub-Saharan Africa. The world is lagging behind in its commitment to end the AIDS epidemic among children. ${ }^{3}$ The pace of progress in reducing new HIV infections among children and expanding access to treatment for children living with HIV has slowed significantly and the global targets set for 2018 have been missed. ${ }^{3}$

In the last two decades, great efforts have been made to reduce the burden of HIV among children by implementing different programmes such as early diagnosis of HIV, increase access to antiretroviral therapy (ART) and prevention of mother-to-child transmission. The ART programme is scaling up to achieve the UNAIDS 90-90-90 targets using a 'Test and Treat' approach. ${ }^{4}$ This has resulted in large numbers of children with HIV enrolling in ART programmes. ${ }^{35}$ More 
than half (54\%) of children living with HIV were receiving lifelong ART in 2018. ${ }^{2}$ The use of ART has substantially reduced HIV-related morbidity and mortality, and increased survival rates for children living with HIV. ${ }^{67}$

Despite this progress, poor treatment outcomes among children with HIV are still a major public health concern in many countries. ${ }^{8-10}$ Children living with HIV and receiving ART continue to experience poor clinical outcomes such as loss to follow-up, treatment failure and death. ${ }^{911}$

Clinical outcomes of children taking ART are affected by several factors including late treatment initiation, treatment regimen, immune status, clinical stage of the disease at the commencement of ART, use of cotrimoxazole preventive therapy, comorbidities (such as TB), and type of HIV (HIV-1 and HIV-2). ${ }^{79} 12$ As ART is a lifelong therapy, the long-term treatment outcome among children is thought to be often poor, and there is no comprehensive evidence that shows the global and regional prevalence of poor treatment outcomes among children with HIV. Hence, quantifying the burden of poor treatment outcomes and identifying the factors associated with poor treatment outcomes is crucial to inform policymakers and service providers, especially in countries where HIV is common. Therefore, this systematic review and meta-analysis will estimate the proportion of poor treatment outcomes and the factors associated with poor treatment outcomes among children with HIV.

\section{Objectives}

The objectives of our study are:

1. To quantify the proportion of poor treatment outcomes among children living with HIV.

2. To identify factors associated with poor treatment outcomes among children living with HIV.

\section{METHODS}

\section{Search strategy}

This systematic review and meta-analysis will adhere to the Preferred Reporting Items for Systematic Reviews and Meta-Analyses guidelines. ${ }^{13}$ Systematic searching will be conducted in PubMed, SCOPUS and Web of Science from 01 Jan 2000 to 28 October 2020 to identify studies that reported treatment outcomes among children with HIV. The search will be limited to studies published in the English language and studies including humans only. We will develop search terms per the Medical Subject Headings thesaurus using a combination of keywords. The full electronic search strategies are provided in box 1. Additionally, a backward and forwards citation search will be undertaken on the reference lists of included studies.

\section{Selection of studies}

All citations identified through our search strategy will be imported into EndNote. After excluding duplicated studies from our EndNote Library, all identified remaining articles will be uploaded into Rayyan. ${ }^{14}$ Screening of papers by title and abstract will be conducted in Rayyan

\section{Box 1 Search strategy}

"HIV Infections" [MeSH] OR "HIV" [Mesh] OR "HIV" [tiab] OR "human immunodeficiency virus" [tiab] OR "AIDS" [tiab] OR "HIV/AIDS" [tiab] AND

Infant[MeSH Terms] OR adolescent[tiab] OR young adult[tiab] OR Child[MeSH Terms] OR child*[tiab] OR infan*[tiab] OR neonate*[tiab] OR newborn*[tiab] OR new born*[tiab] OR baby[tiab] OR babies[tiab] OR toddler*[tiab] OR boy[tiab] OR boys[tiab] OR girl*[tiab] OR pediatric[tiab] OR "paediatric"[tiab] OR prepubescen*[tiab] OR prepuberty*[tiab] AND

Mortality [MeSH Terms] OR "survival rate" [Mesh Terms] OR prognosis [MeSH Terms] OR mortality[tiab] OR death[tiab] OR fatal[tiab] OR "survival rate" [tiab] OR "death rate" [tiab] OR prognosis[tiab] OR "lost" OR "treatment outcomes" [tiab]

MeSH, Medical Subject Headings.

by two independent reviewers (KAA1 and KAA2). If the title and abstract of a paper appear relevant, the full text of the paper will be reviewed. Disagreement on the inclusion or exclusion of selected papers will be resolved through discussion and consensus. The reviewers will conduct a pilot exercise for both title/abstract screening and full-text screening on 10 randomly selected articles. We will include studies based on the following eligibility criteria.

\section{Participants}

Studies examining poor treatment outcomes in children (under the age of 18 years) living with HIV who take ART at least for 6 months. Studies that report results separately for children and adults will be also included.

\section{Outcomes}

Studies explicitly reporting on poor treatment outcomes in children living with HIV. Poor treatment outcomes that include loss to follow-up, treatment failure and death are the primary outcomes of this systematic review. Adverse events and poor adherence to HAART use will be also included as a secondary outcome. Treatment failure will be defined as per the definition by the study authors. We will also use our standard definition for poor treatment adherence (eg, $<80 \%$ adherence).

\section{Study designs}

Observational studies, such as retrospective cohort, prospective cohort, case-control and cross-sectional studies, as well as interventional studies written in the English language, will be included.

\section{Exclusion criteria}

We will exclude case reports, case series, correspondence and abstracts without the full text. We will contact the authors of papers by email if relevant information is missing or unclear. We will send a total of two emails to the corresponding author, 2 weeks apart. If there is no response from the corresponding author, we will then decide if the paper can be included in the review or not. We will make these decisions by consensus. 


\section{Data extraction}

Data will be extracted from eligible studies by two independent reviewers (KAA1 and KAA2), and information will be collated in a Microsoft Excel V.2016 spreadsheet. The data extraction spreadsheet will be piloted on 10 randomly selected papers. We will collect data on the outcomes of our study from each individual paper (ie, lost to follow-up, treatment failure, death, adverse events and poor adherence). We will record treatment outcomes of children living with HIV as prevalence, cumulative incidence, incidence density or incidence proportion as reported in the individual papers. We will also record relative risks or ORs to calculate relative risk compared with controls if data are available. We will also extract the following data from each paper: name of the first author, year of publication, a country in which the study was conducted, study design, number of included study participants, participation rate, baseline socioeconomic indicators (such as mean family income level and percentage of people with low income), demographic factors (including mean age in years and percentage of male patients), HIV diagnostic method, percentage of patients with $\mathrm{TB}$ infection and percentage of patients with other illnesses at baseline (if known). We will also collect data on the mean number of drugs provided to patients with HIV, the mean duration of HIV treatment in months, treatment regimen and drug-resistance pattern. Additionally, all variables significantly associated with poor treatment outcomes will be recorded to assess the factors associated with the pooled proportion of poor treatment outcomes.

\section{Quality assessment}

Two authors (KAA1 and KAA2) will assess the methodological quality of the included studies, including the risk of bias in the selection of the study groups (ie, people diagnosed with HIV) and outcome ascertainment using the Newcastle-Ottawa Scale. The quality assessment tool will be piloted on 10 randomly selected papers to increase agreement between the two reveries.

\section{STATISTICAL ANALYSIS}

\section{Data analysis}

A random-effects meta-analysis will be used to obtain pooled estimates of the proportion of poor treatment outcomes when two or more studies are available. When meta-analysis is not possible, a narrative synthesis will be used to describe the outcomes of the study. The randomeffects model will assume that there is a heterogeneity of true-effect sizes of poor treatment outcomes in children living with HIV.

\section{Assessment of heterogeneity and reporting biases}

The heterogeneity between studies will be checked visually by using forest plots and quantitatively measured by the index of heterogeneity ( $\mathrm{I}^{2}$ and its $\mathrm{CI}$ ), and Cochran Q-statistics test. Heterogeneity between studies will be considered low, moderate and high when $\mathrm{I}^{2}$ values are below $25 \%$, between $25 \%$ and $75 \%$, and above $75 \%$, respectively $^{15}$; and for Q-statistics heterogeneity will be assumed at a $\mathrm{p}$ value less than 0.05 . Small study bias or publication bias will be checked by using funnel plots (visual) or Egger's test (statistical). A p value of $<0.05$ will be considered as indicative of publication bias with the Egger's test.

\section{Subgroup analysis}

We will conduct a subgroup analysis to assess the effects of each study characteristic on the primary outcomes of the study. A stratified analysis will be performed by the study setting, study design, demographic factors such as sex, treatment regimens of HIV and comorbidities such as TB infection.

\section{Patient and public involvement \\ No patient will be involved in the study.}

\section{Ethics and dissemination}

Ethical approval will not be required for this study as it will be based on de-identified, aggregate published data. The final report of this review will be disseminated through publication in a peer-reviewed scientific journal and will be presented at relevant conferences.

\section{DISCUSSION}

Due to the lifelong treatment schedule, the treatment of HIV is more challenging in children and often results in poor treatment outcomes. ${ }^{16-20}$ Strengthening healthcare services to ensure better treatment outcomes in HIV-infected children is essential. ${ }^{21-23}$ Although several studies have been conducted to assess the clinical burden and treatment outcomes of HIV in adults, ${ }^{24-26}$ treatment outcomes are poorly understood among children. ${ }^{27}$ This systematic review and meta-analysis will provide pooled estimates of poor treatment outcomes among children who were diagnosed with HIV and enrolled for treatment with highly active ART. It will also identify the main risk factors for poor treatment outcomes of children living with HIV. Identifying the main risk factors for poor treatment outcomes using a comprehensive systematic review and meta-analysis will provide empirical evidence necessary for clinicians and health experts to better understand the risk factors, policy implications, future research needs, and programming priorities for the treatment, care and follow-up of children with HIV.

Contributors KAA1 and KAA2 conceived of the study, developed the search strategy and drafted the protocol. Both authors critically revised the manuscript for methodological and intellectual content, and have read and approved the final manuscript.

Funding The authors have not declared a specific grant for this research from any funding agency in the public, commercial or not-for-profit sectors.

Competing interests None declared.

Patient and public involvement Patients and/or the public were not involved in the design, or conduct, or reporting, or dissemination plans of this research. 
Patient consent for publication Not required.

Provenance and peer review Not commissioned; externally peer reviewed.

Open access This is an open access article distributed in accordance with the Creative Commons Attribution Non Commercial (CC BY-NC 4.0) license, which permits others to distribute, remix, adapt, build upon this work non-commercially, and license their derivative works on different terms, provided the original work is properly cited, appropriate credit is given, any changes made indicated, and the use is non-commercial. See: http://creativecommons.org/licenses/by-nc/4.0/.

ORCID iDs

Kendalem Asmare Atalell http://orcid.org/0000-0003-1127-3592

Kefyalew Addis Alene http://orcid.org/0000-0002-1904-4682

\section{REFERENCES}

1 HIV/AIDS JUNPo. Unaids data 2017. 2017. Geneva: Switzerland: UNAIDS, 2018.

2 UNAIDS. Global HIV \& AIDS statistics - 2019 fact sheet, 2019.

3 WHO. UNAIDS calls on countries to accelerate efforts and close service gaps to end the AIDS epidemic among children and adolescents, 2019

4 Euvrard J, Schulz T, Hilderbrand K, et al. How accurately do routinely reported HIV viral load suppression proportions reflect progress towards the 90-90-90 target in the population on antiretroviral treatment in Khayelitsha, South Africa? S Afr Med J 2019;109:174-7.

5 Nachega JB, Adetokunboh O, Uthman OA, et al. Community-Based interventions to improve and sustain antiretroviral therapy adherence, retention in HIV care and clinical outcomes in low- and middleincome countries for achieving the UNAIDS 90-90-90 targets. Curr HIVIAIDS Rep 2016;13:241-55.

6 Berendam SJ, Nelson AN, Goswami R, et al. Pediatric HIV: the potential of immune therapeutics to achieve viral remission and functional cure. Curr HIVIAIDS Rep 2020;17:237-48.

7 Hansudewechakul R, Sirisanthana V, Kurniati N, et al. Antiretroviral therapy outcomes of HIV-infected children in the treat Asia pediatric HIV observational database. J Acquir Immune Defic Syndr 2010;55:503-9.

8 Frohoff C, Moodley M, Fairlie L, et al. Antiretroviral therapy outcomes in HIV-infected children after adjusting protease inhibitor dosing during tuberculosis treatment. PLoS One 2011;6:e17273.

9 Poor clinical outcomes for HIV infected children on antiretroviral therapy in rural Mozambique: need for quality improvement and continuing program/community development in PEPFAR2012XIX International AIDS Conference Washington, DC

10 Okomo U, Togun T, Oko F, et al. Treatment outcomes among HIV-1 and HIV-2 infected children initiating antiretroviral therapy in a concentrated low prevalence setting in West Africa. BMC Pediatr 2012;12:95.

11 Resino S, Resino R, Maria Bellón J, et al. Clinical outcomes improve with highly active antiretroviral therapy in vertically HIV type-1infected children. Clin Infect Dis 2006;43:243-52.
12 Mutanga JN, Mutembo S, Ezeamama AE, et al. Long-Term survival outcomes of HIV infected children receiving antiretroviral therapy: an observational study from Zambia (2003-2015). BMC Public Health 2019:19:115.

13 Moher D, Liberati A, Tetzlaff J, et al. Preferred reporting items for systematic reviews and meta-analyses: the PRISMA statement. Ann Intern Med 2009;151:264-9.

14 Ouzzani M, Hammady H, Fedorowicz Z, et al. Rayyan-a web and mobile APP for systematic reviews. Syst Rev 2016;5:210.

15 Higgins JPT, Thompson SG. Quantifying heterogeneity in a metaanalysis. Stat Med 2002;21:1539-58.

16 Kabue MM, Buck WC, Wanless SR, et al. Mortality and clinical outcomes in HIV-infected children on antiretroviral therapy in Malawi, Lesotho, and Swaziland. Pediatrics 2012;130:e591-9.

17 Fatti G, Bock P, Eley B. Temporal trends in baseline characteristics and treatment outcomes of children starting antiretroviral treatment: an analysis in four provinces in South Africa, 2004-2009 2011;58:e60-7.

18 Davies M-A, Keiser O, Eley B. Outcomes of the South African national antiretroviral treatment programme for children: the leDEA Southern Africa collaboration. 99, 2009

19 Nyandiko WM, Ayaya S, Nabakwe E, et al. Outcomes of HIV-infected orphaned and non-orphaned children on antiretroviral therapy in Western Kenya. J Acquir Immune Defic Syndr 2006;43:418-25.

20 O'Brien DP, Sauvageot D, Olson D. Treatment outcomes stratified by baseline immunological status among young children receiving nonnucleoside reverse-transcriptase inhibitor-based antiretroviral therapy in resource-limited settings. , 2007: 44, 1245-8.

21 Ginossar T, Van Meter L, Ali Shah SF, et al. Early impact of the patient protection and Affordable care act on people living with HIV: a systematic review. J Assoc Nurses AIDS Care 2019;30:259-69.

22 Tran BX, Nguyen QN, Nguyen LH. Factors associated with improved HIVIAIDS treatment outcomes: comparing two major art service delivery models in Vietnam, 2019: 1-9.

23 Busza J, Dauya E, Bandason T, et al. The role of community health workers in improving HIV treatment outcomes in children: lessons learned from the ZENITH trial in Zimbabwe. Health Policy Plan 2018;33:328-34.

24 Cioe PA, Gordon RE, Williams DM. The effect of increased physical activity on symptom burden in older persons living with HIV, 2019: 1-7.

25 Mabweazara SZ, Ley C, Leach LL, LLJAJoAR L. Physical activity, social support and socio-economic status amongst persons living with HIV and AIDS: a review. African Journal of AIDS Research 2018;17:203-12.

26 Black J, Bond MA, Hawkins R. Test of a clinical model of poor physical health and suicide: the role of depression, psychosocial stress, interpersonal conflict, and panic, 2019.

27 Mossoro-Kpinde CD, Gody J-C, Mboumba Bouassa R-S, et al. Escalating and sustained immunovirological dissociation among antiretroviral drug-experienced perinatally human immunodeficiency virus-1-infected children and adolescents living in the central African Republic: a STROBE-compliant study. Medicine 2020;99:e19978. 